\title{
Picturing peripheral acuity
}

\section{Stuart Anstis}

Department of Psychology, University of California at San Diego, La Jolla, CA 92093-0109, USA; e-mail: sanstis@ucsd.edu

Received 1 December 1997, in revised form 9 May 1998

\begin{abstract}
The grain of the retina becomes progressively coarser from the fovea to the periphery. This is caused by the decreasing number of retinal receptive fields and decreasing amount of cortex devoted to each degree of visual field (= cortical magnification factor) as one goes into the periphery. We simulate this with a picture that is progressively blurred towards its edges; when strictly fixated at its centre it looks equally sharp all over.
\end{abstract}

Foveal acuity is far better than peripheral acuity, since the structure of the retina and cortex is designed to favour foveal in preference to peripheral stimuli. The visual field is mapped topographically on the surface of the striate cortex in humans; the projection is large for the central visual field and is progressively compressed toward the periphery (Mora et al 1989; Sereno et al 1995). Visual acuity decreases with distance from the fovea in proportion to the estimated cortical magnification factor, CMF, defined as the number of millimetres on the cortex to which one degree on the retina projects, and thus its reciprocal is the number of degrees of visual angle per millimetre of cortex. The reciprocal of the CMF increases linearly with eccentricity, from about $0.15 \mathrm{deg} \mathrm{mm} \mathrm{mm}^{-1}$ at the fovea to $1.5 \mathrm{deg} \mathrm{mm}^{-1}$ at an eccentricity of $20 \mathrm{deg}$ (Hubel and Wiesel 1974, macaque). There is additional magnification of the central visual field as one goes from the retinal ganglion cells to the cerebral cortex (Myerson et al 1977, owl monkey; Van Essen et al 1984, macaque). The eventual cortical magnification of the central retina is such that about $25 \%$ of the striate cortex is devoted to processing the central $2.5 \mathrm{deg}$ of the visual scene (De Valois and De Valois 1988, page 95). The cortical map reflects roughly a complex logarithmic transform of the retinal spatial mapping (Schwartz 1980).

If a stimulus is magnified at peripheral locations in proportion to the reciprocal of the $\mathrm{CMF}$, it becomes equally resolvable across the visual field. This scaling procedure (M-scaling) normalises visual field loci irrespective of eccentricity (Daniel and Whitteridge 1961, monkey; Johnston and Wright 1983, human; Pointer 1986). Many psychophysical studies in humans have shown that, when stimuli are M-scaled, the near periphery resembles foveal vision. This has been shown to hold true for sensitivity to stationary, moving, and flickering gratings (Koenderink et al 1978; Rovamo et al 1978; Rovamo and Virsu 1979; Virsu and Rovamo 1979; Virsu et al 1982; Bijl et al 1992); the contrast sensitivity function, which shifts to lower spatial frequencies with increasing eccentricity while keeping about the same total bandwidth (Kelly 1984); Vernier acuity (Levi et al 1985); discrimination of second-order textures (Saarinen et al 1987); wavelength discrimination, at least over eccentricities from 8 to $80 \mathrm{deg}$ (van Esch et al 1984); the size of Panum's fusional area (Hampton and Kertesz 1983); and the perceptive-field size in the Westheimer paradigm (Westheimer 1967; Ransom-Hogg and Spillmann 1980). It is also true for sensitivity to small vertical displacements (Haines and Kiefel 1988), and detection of coherent movement in peripherally viewed random-dot patterns (van de Grind et al 1983). Johnston and Wright (1983) and Wright and Johnston (1985) found that both the lower threshold of motion and adaptation to 
motion were uniform for $\mathrm{M}$-scaled stimuli and were related to the velocity of the cortical image. The perceptive fields for a ramp aftereffect from dimming or brightening light were found to be M-scaled (Anstis and Harris 1987). I published an eye chart with small letters near the middle and progressively larger letters further out, reflecting the coarsening of the retinal grain with eccentricity (Anstis 1974). Grüsser (1995) demonstrated that migraine fortifications are correlated with M-scaling, and Teuber et al (1960) pointed out that brain penetration by a bullet results in a small foveal scotoma, but a much larger peripheral scotoma, in accordance with inverse optics. M-scaling has been incorporated into visual modelling (eg Watson 1983; Wilson et al 1990).

M-scaling is not found universally. Some visual performances are independent of eccentricity, for instance spectral sensitivity as measured by flicker photometry (van Esch et al 1984). Adaptation to flicker falls off with eccentricity, but more slowly than one would expect from M-scaling (Anstis 1996). On the other hand, performance on other tasks falls off with eccentricity, even after the stimuli have been M-scaled. Examples are the detection of circular disks (Bijl et al 1992), the detectability of mirror symmetry in line-segment patterns (Saarinen 1987) and in random-dot patterns (Saarinen 1988), and information about the local quality of spatial structures (Jamar et al 1984). Meinecke and Kehrer (1994) found that performance in a texture segmentation task peaked at an eccentricity of $6 \mathrm{deg}$. It should be noted that different psychophysical tasks often show very different cortical magnification factors, suggesting vastly different retina-tocortex mappings. For example, Vernier acuity falls off with eccentricity much faster than grating acuity (Rovamo et al 1993; Schwartz 1994, table 1).

Some visual performances, such as critical flicker frequency (CFF) and increase of grating acuity with luminance, require two separate steps to make them similar at all eccentricities: (1) M-scaling, and (2) 'F-scaling', in which retinal illuminance is multiplied by Ricco's area to express luminance in terms of photopic luminous flux (Rovamo and Raninen 1984, 1990; Raninen and Rovamo 1986). These authors conclude that, with constant stimulus area and luminance, the variation of CFF with visual field location results from two counteracting factors: the decrease of ganglion cell density toward the retinal periphery, which tends to reduce CFF, and the increase of receptive field size toward the retinal periphery, which tends to enhance CFF. In photopic vision, CFF falls off monotonically toward the periphery, in agreement with cone density, whereas in scotopic vision it peaks between 12 and 18 deg in agreement with rod density. Despite these various exceptions, the general efficacy of $\mathrm{M}$-scaling attests that central and peripheral vision are qualitatively similar in spatiotemporal visual performance.

A well-known example of this is the reduction of acuity with increasing eccentricity. Whereas a newspaper is legible in foveal vision, only headlines are large enough to be resolved in the periphery. Figure 1 simulates this falling off of resolution as one goes from the fovea to the periphery. The grey-scale picture in figure la has been blurred in figure $1 \mathrm{~b}$ by applying radial blur $(\operatorname{spin}=1$, zoom $=4$ ) in Adobe Photoshop, to produce a picture which keeps its original sharpness at the centre but is progressively blurred toward the periphery. It is important that the reader should at all times strictly fixate the centre of the picture, just above the head of the man in the black jacket. The blur function in figure $1 b$ has been exaggerated for purposes of exposition, but if it were not for this then figure $1 \mathrm{~b}$ would look equally sharp all over because the progressive blur in the stimulus would match the progressive neural blur in the eye. The amount of blurring in the stimulus gives a rough idea of the amount of neural blurring that occurs in peripheral vision, of which we are seldom aware. Ideally, an alternation of the superimposed sharp and blurred pictures (figures $1 \mathrm{a}$ and 1b), during strict fixation of the centre, would yield no perceptible change in the picture, which might be said to show that the two images are metameric. If one were able to design a sharp high-frequency cutoff filter with the correct spatial rate of blur, then all details across 


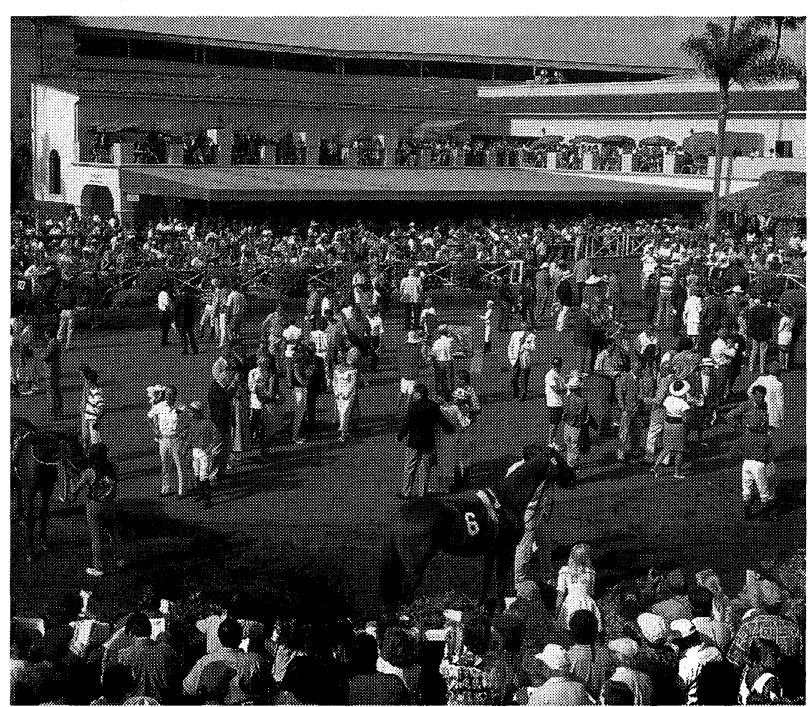

(a)

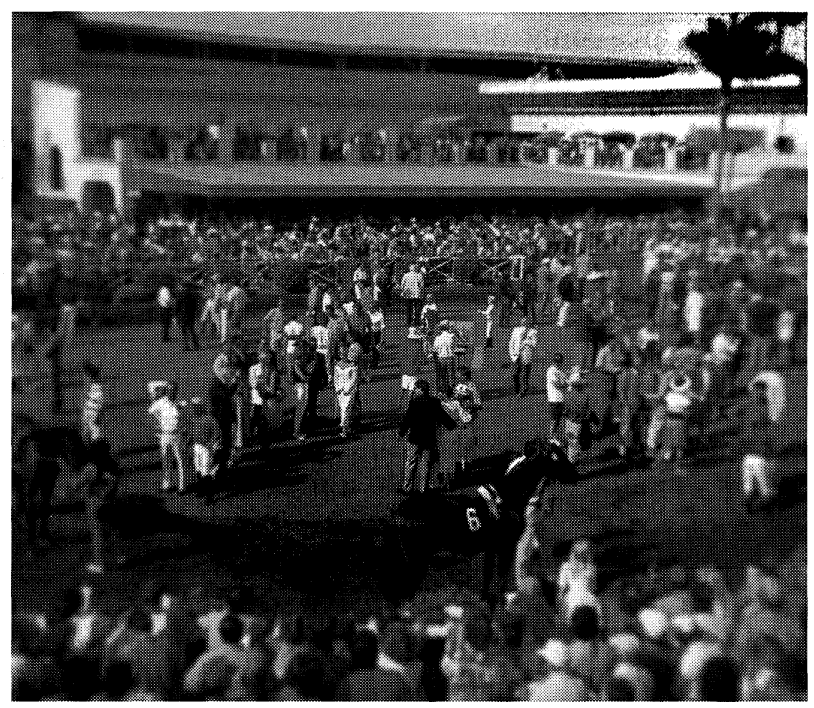

(b)
Figure 1. The picture (a) is progressively blurred from the centre outwards (b), simulating the progressive loss of acuity with eccentricity.

the picture will lie just below the Nyquist frequency of the part of the retina on which it falls, and then the two pictures would be indistinguishable. Oddly enough, the viewing distance makes no difference, because bringing the picture closer to one's eyes makes the details get larger but also moves them further out into the periphery, where the loss in acuity exactly balances out the gain in size. There have been some good studies of blur discrimination (Watt and Morgan 1983; Hess et al 1989; Levi and Klein 1990; Westheimer 1991; Mather 1996) but here we are studying the non-discrimination of blur.

Why is the visual system organised with these varying levels of acuity? We calculate that it achieves considerable data compression which can reduce the bandwidth of a picture transmitted to the brain by up to $97 \%$. Rounding off some numbers for humans, taken from Curcio et al (Curcio and Allen 1990; Curcio et al 1990), there are 1000000 ganglion cells spread unequally across the total retinal area of $1000 \mathrm{~mm}^{2}$. In the central fovea the peak density of ganglion cells is 35000 cells $\mathrm{mm}^{-2}$, with at least two ganglion cells connected to every cone in the central fovea, perhaps one ON-ganglion and one 
OFF-ganglion. As one proceeds further into the periphery, of course, the ganglion receptive fields get larger, and more and more photoreceptors have to share each ganglion cell. Curcio et al bring out the extreme inequality of the distribution of retinal ganglion cells by pointing out that the change in the number of ganglion cells from fovea to periphery is in the order of $1000: 1$, and that $50 \%$ of the ganglion cells lie within $16 \mathrm{deg}$ of the foveal centre, a region that comprises only $7 \%$ of the total area. Figure 1 ignores several known nonuniformities, such as a slight favouring of the lower visual field (superior retina), which at eccentricities greater than 2.5 deg has $12 \%$ more visual cortex (Van Essen et al 1984, macaque), and at eccentricities greater than 15 deg has 60\% more retinal ganglion cells (Curcio and Allen 1990, human), compared to the upper visual field (inferior retina). There is a much greater favouring of the temporal visual field (nasal retina), which at eccentricities greater than 15 deg has $300 \%$ more retinal ganglion cells (Curcio and Allen 1990), compared to the nasal visual field (temporal retina).

The advantages of the real visual system can be brought out by comparing it with two imaginary ones. If all the existing 1000000 ganglion cells were to be packed in together at the peak density of 35000 cells $\mathrm{mm}^{-2}$ without increasing their number, so that the whole retina shrank down until it had the maximum acuity found in the central fovea, then the area of the retina would shrink to $3 \%$ of its existing size and the field of view would contract to an area only $20 \mathrm{deg} \times 20 \mathrm{deg}$. This may sound reasonably large - after all, it extends out to the inner edge of the blind spot—but although this kind of high-acuity tunnel vision might suit a watchmaker, it would be of little use to an organism that relies upon peripheral vision to guide locomotion and to spot approaching predators.

Conversely, if one were to raise the acuity of the whole retina up to that obtainable in the central fovea without reducing the size of the retinal field, the number of ganglion cells would have to increase from 1000000 to 35000000 . The diameter of the retina would increase sixfold and the eyeball would swell up to be nearly as big as one's head! The optic nerves would thicken up and the brain would have to expand correspondingly. In fact Schwartz (1994) calculated that there is approximately a $10000: 1$ compression provided by the declining magnification toward the periphery, and following this rationale he arrives at a hypothetical weight of 5000-30000 lb for the human brain if the same high spatial resolution as in the fovea is assumed for the entire retina. Since the existing brain is only $2 \%$ of the body weight but commands $20 \%$ of the blood supply, any expansion of the brain would require a larger heart, and the system would quickly become unmanageable. The tapered acuity of the visual system is a neat compromise between the two undesirable extremes of tunnel vision on the one hand and an unacceptably huge visual system on the other. However, it involves further costs in that the retina must be steerable. The periphery can be likened to the largefield low-resolution finder telescope that astronomers use to acquire targets and steer the main small-field high-acuity telescope-the fovea-towards these targets. The visual system uses saccadic eye movements triggered by sudden peripheral changes to acquire targets with the fovea. The peripheral retina is very sensitive to motion, and highly accurate saccades are needed to bring the fovea on to the 'where', adding information to the 'what'. Presumably the whole elaborate mechanism of eye movements is an alternative that is computationally and metabolically cheaper than the imaginary large, high-acuity retina which it replaces. Even insect eyes, which have evolved along entirely different design principles, contain regions of different acuity. There seem to be two kinds of acute zones in insect eyes, one concerned with forward flight and the other with the detection and capture of other insects (Land and Eckert 1985). Many insects, together with cuttlefish and crabs, have also evolved a pattern of fixations and saccades similar to vertebrates, together with reflex compensation for body rotation. In some 
flying insects the same eye movements occur, but, unencumbered by contact with the ground, it is now the whole body that makes the saccades and fixations, or in some cases tracks a target (Land 1995).

This method applies to other sensory systems too. The receptive fields of skin receptors in the arm are smallest at the fingertips and progressively larger up the arm, and the cortical magnification factor for the fingertips is 100 times as great as for the trunk (see Mountcastle 1984). A baby whose wrist touches an object, which he then attempts to grab with his high-acuity fingers, is using a similar technique. If his cheek touches a nipple, he steers his high-acuity lips toward the food source. In both cases the fingertips and lips are like the fovea of the arm or face, and his grasping movements are analogous to saccadic eye movements.

With strict central fixation, the progressive blurring of the picture, if correctly matched to the eye, would not be detectable, although turning the eyes toward the edge of the picture would immediately reveal it. A large page of text, fixated at its centre, creates the illusion of being equally sharp and equally legible all over. It is only when one attends carefully to the peripheral parts of the page, while maintaining strict fixation, that one becomes aware that these parts are illegible. And this act of shifting attention is itself no easy or natural task, since attention seems to gravitate to the fovea rather as a marble tends to roll down to the bottom of a bowl.

Why does the whole visual field normally look equally sharp all over, when there is clearly an enormous degradation of the visible detail in peripheral vision? This is an illposed question. After all, if our acuity were 1000 times better than it is, we could throw away our microscopes, yet our 'limited' foveal acuity which prevents us from seeing bacteria with the naked eye never looks like any kind of subjective blur! The same applies to our limited peripheral acuity. A channel cannot signal anything about stimuli to which it is not tuned, so peripheral retinal channels must remain silent in the presence-or absence-of high spatial frequencies to which they are blind. This is not to rule out the possible existence of blur constancy across the retina, which might be investigated by matching blurred edges seen in the periphery with an edge of adjustable blur seen in the fovea.

The cortical magnification factor can also be plotted as a barrel distortion, defined as a magnification which is greater at the centre than at the edges of a picture, as is the cortical image in V1. Figure 2 shows the barrel distortion of the retinal image (figure 2a) that is introduced by the retinal ganglion cells [figure $2 \mathrm{~b}$; circles interpolated from Curcio and Allen (1990), figure 7] and in cortical area V1 [figure 2c; circles interpolated from a 2-deoxyglucose anatomical study by Tootell et al (1982)].

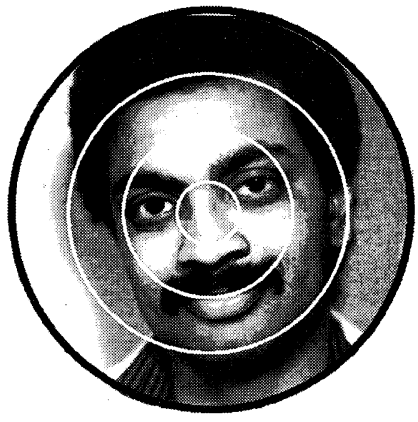

(a) Retinal image

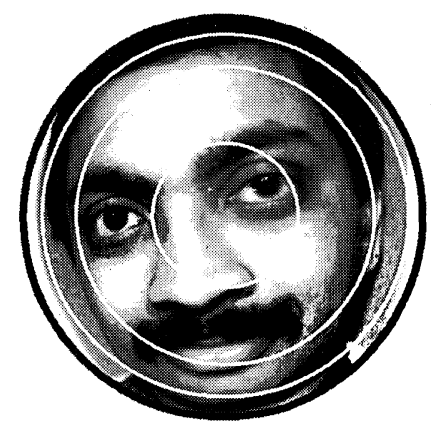

(b) Ganglion layer

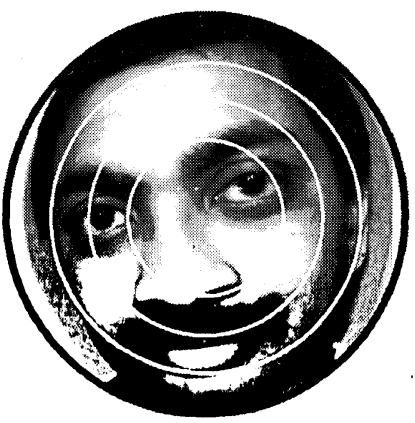

(c) V1

Figure 2. The retinal image (a) becomes barrel distorted by the magnification factor in the retinal ganglion layer [(b), after Curcio and Allen (1990), human retina] and the cortical area V1 [(c), after Tootell et al (1982), macaque cortex]. 
The question why one does not perceive the barrel distortion of one's own cortical image is also ill-posed and falls into the homunculus fallacy. The brain does not know what shape it is, and there is no homunculus looking at the size and shape of the cortical image. But the question can be refined by asking how one achieves size constancy across the retinal surface. How can one see a line or a circle as maintaining a constant size and shape without $\mathrm{M}$-scaling when it moves across the retina, stimulating ever different numbers of cells in each retinal region? There must be some metric that is independent of the number of cells. Even a small failure in this constancy process should lead to perceptible size distortions. Such distortions have been noted by Helmholtz (1910/1962). He noted that a dark door seen in the far periphery to the left or right of fixation looks apparently taller and narrower than it is. (Such distortions are easiest to see in small, isolated stimuli. I have noticed that a $\operatorname{dim} 0.5 \mathrm{~cm}$ spot flashed up repeatedly in a perimeter looks decidedly smaller and sharper when viewed in the periphery compared to parafoveally.) Again, if one finds three well-separated stars in the night sky that lie in a straight line, they will be seen veridically if one fixates the central star, but if one moves one's fixation to left or right the line of stars looks concave toward the new fixation point, the concavity being more and more pronounced, the further the point of fixation is from the row of stars. Thus when the eye looks steadily in one direction, a great circle on the celestial sphere will not appear straight unless it passes through the point of fixation; otherwise it will appear curved, concave toward that point. These phenomena may indicate a small, partial failure to compensate for the barrel distortion in the cortical image. Helmholtz goes on to present a checkerboard composed of hyperbolas (figure 3). This is like a square checkerboard to which strong pincushion distortion has been applied, defined as a magnification which is greater at the edges than in the middle (the inverse of barrel distortion). When this is viewed from very close up the checkerboard does look square. Helmholtz's account is for once not entirely clear (at least to this reader), but an additional factor here is that the checkerboard lies on a plane sheet of paper very near the eye, which may simply be projecting a square checkerboard onto the roughly spherical surface of the retina.

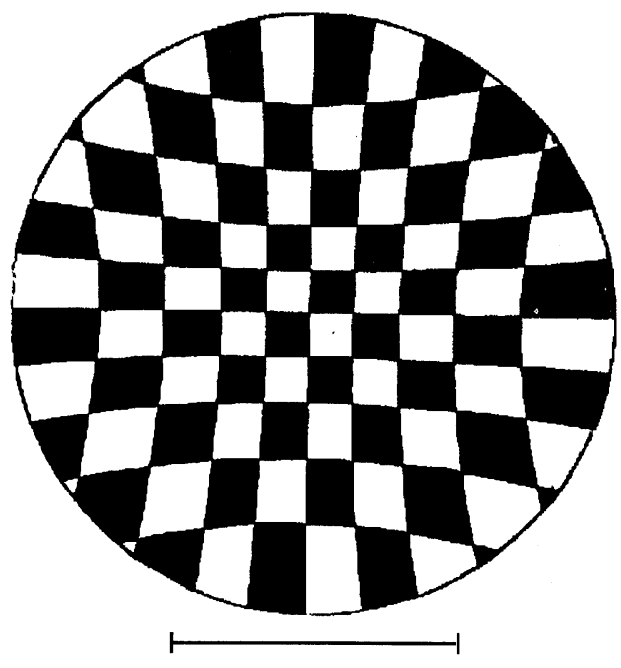

Figure 3. This pincushion-distorted checkerboard should be viewed from a distance as long as the horizontal bar. It will then look like a square checkerboard (Helmholtz 1910/1962). 
Acknowledgements. Supported by NIH Grant EY-10241. Thanks to David Peterzell, Harvey Smallman, and two anonymous referees for comments on the manuscript.

\section{References}

Anstis S M, 1974 "A chart demonstrating variations in acuity with retinal position" Vision Research $14589-592$

Anstis S M, 1996 "Adaptation to peripheral flicker" Vision Research 36 3479-3485

Anstis S M, Harris J P, 1987 "Magnification factor for adaptation of a visual transient mechanism" Journal of the Optical Society of America A 4 1688-1698

Bijl P, Koenderink J J, Kappers A M, 1992 "Deviations from strict M scaling" Journal of the Optical Society of America A $91233-1239$

Cowey A, Rolls E T, 1974 "Human cortical magnification factor and its relation to visual acuity" Experimental Brain Research 21 447-454

Curcio C, Allen K A, 1990 "Topography of ganglion cells in human retina" Journal of Comparative Neurology 300 5-25

Curcio C, Sloan K R, Kalina R E, Henderson A, 1990 "Human photoreceptor topograph" Journal of Comparative Neurology 292 497-523

Daniel M, Whitteridge D, 1961 "The representation of the visual field on the cerebral cortex in monkeys" Journal of Physiology (London) 159 203-221

De Valois R L, De Valois K K, 1988 Spatial Vision (New York: Oxford University Press)

Dobelle W H, Turkel J, Henderson D C, Evans J R, 1974 "Mapping the representation of the visual field by electrical stimulation of human visual cortex" American Journal of Ophthalmology 88 $727-735$

Drasdo N, 1977 "The neural representation of visual space" Nature (London) 266 554-555

Drasdo N, 1989 "Receptive field densities of the ganglion cells of the human retina" Vision Research $29985-988$

Drasdo N, Fowler C W, 1974 "Non-linear projection of the retinal image in a wide-angle schematic eye" British Journal of Ophthalmology 58 709-714

Esch J A van, Koldenhof E E, Doorn A J van, Koenderink J J, 1984 "Spectral sensitivity and wavelength discrimination of the human peripheral visual field" Journal of the Optical Society of America A $1443-450$

Fox P T, Miezin F M, Allman J M, Van Essen D C, Raichle M E, 1987 "Retinotopic organization of human visual cortex mapped with positron-emission tomography" Journal of Neuroscience 7 $913-922$

Grind W A van de, Doorn A J van, Koenderink J J, 1983 "Detection of coherent movement in peripherally viewed random-dot patterns" Journal of the Optical Society of America 73 $1674-1683$

Grüsser O-J, 1995 "Migraine phosphenes and the retino-cortical magnification factor" Vision Research $351125-1134$

Haines R F, Kiefel S M, 1988 "Vertical displacement threshold sensitivity along the horizontal meridian as a function of stimulus rate, duration, and length" Aviation, Space, \& Environmental Medicine $59321-329$

Hampton D R, Kertesz A E, 1983 "The extent of Panum's area and the human cortical magnification factor" Perception $12161-165$

Helmholtz H von, 1910/1962 Treatise on Physiological Optics volume 3 (New York: Dover, 1962); English translation by J P C Southall for the Optical Society of America (1925) from the 3rd German edition of Handbuch der physiologischen Optik (Hamburg: Voss, 1910), figure 22, page 181

Hess R F, Pointer J S, Watt R J, 1989 "How are spatial filters used in fovea and parafovea?" Journal of the Optical Society of America A $6329-339$

Hubel D H, Wiesel T N, 1974 "Uniformity of monkey striate cortex: A parallel relationship between field size, scatter and magnification factor" Journal of Comparative Neurology 158 296-306

Jamar J H, Kwakman L F, Koenderink J J, 1984 "The sensitivity of the peripheral visual system to amplitude modulation and frequency modulation of sine-wave patterns" Vision Research $24243-249$

Johnston A, Wright M J, 1983 "Visual motion and cortical velocity" Nature (London) 304 436-438

Kelly D H, 1984 "Retinal inhomogeneity. I. Spatiotemporal contrast sensitivity" Journal of the Optical Society of America A $1107-113$

Koenderink J J, Bouman M A, Bueno de Mesquita A E, Slappendel S, 1978 "Perimetry of contrast detection thresholds of moving spatial sine-wave patterns: III. The target extent as a sensitivity controlling parameter" Journal of the Optical Society of America 68 854-860 
Land M F, 1995 "The functions of eye movements in animals remote from man", in Eye Movement Research Ed. J M Findlay (Amsterdam: Elsevier Science) pp 63-76

Land M F, Eckert H, 1985 "Maps of the acute zones of fly eyes" Journal of Comparative Physiology 156 525-538Levi D M, Klein S A, 1990 "Equivalent intrinsic blur in spatial vision" Vision Research $301971-1993$

Levi D M, Klein S A, Aitsebaomo A P, 1985 "Vernier acuity, crowding and cortical magnification" Vision Research $25963-977$

Mather G, 1996 "Image blur as a pictorial depth cue" Proceedings of the Royal Society of London, Series B $263169-171$

Meinecke C, Kehrer I, 1994 "Peripheral and foveal segmentation of angle texture" Perception \& Psychophysics 56 326-334

Mora B N, Carman G J, Allman J M, 1989 "In vivo functional localization of the human visual cortex using positron emission tomography and magnetic resonance imaging" Trends in Neuroscience $12282-286$

Mountcastle V, 1984 "Central nervous mechanisms in mechanoreceptive sensibility", in Handbook of Physiology Ed. I Darian-Smith: Section 1: The Nervous System Volume III. Sensory Processes Part 2 (Bethesda, MD: American Physiological Society) pp 789-878

Myerson J, Manis P B, Miezin F M, Allman J M, 1977 "Magnification in striate cortex and retinal ganglion cell layer of owl monkey: A quantitative comparison" Science 198 855-857

Pointer J S, 1986 "Visual representation at the cerebral cortex: Qualitative and quantitative aspects" Ophthalmic \& Physiological Optics $6171-175$

Raninen A, Rovamo J, 1986 "Perimetry of critical flicker frequency in human rod and cone vision" Vision Research $261249-1255$

Ransom-Hogg A, Spillmann L, 1980 "Perceptive field size in fovea and periphery of the light- and dark-adapted retina" Vision Research 20221 - 228

Rovamo J, Mäkelä P, Whitaker D, 1993 "Models of the visual cortex on the basis of psychophysical observations", in Functional Organisation of the Human Visual Cortex Eds B Gulyas, D Ottoson, P E Roland (Oxford: Pergamon Press)

Rovamo J, Raninen A, 1984 "Critical flicker frequency and M-scaling of stimulus size and retinal illuminance" Vision Research 241127 - 1131

Rovamo J, Raninen A, 1990 "Cortical acuity and the luminous flux collected by retinal ganglion cells at various eccentricities in human rod and cone vision" Vision Research 30 11-21

Rovamo J, Virsu V, 1979 "An estimation and application of the human cortical magnification factor" Experimental Brain Research 37 495-510

Rovamo J, Virsu V, Näsänen R, 1978 "Cortical magnification factor predicts the photopic sensitivity of peripheral vision" Nature (London) 271 54-56

Saarinen J, 1987 "Perception of positional relationships between line segments in eccentric vision" Perception $16583-591$

Saarinen J, 1988 "Detection of mirror symmetry in random-dot patterns at different eccentricities" Vision Research $28755-759$

Saarinen J, Rovamo J, Virsu V, 1987 "Texture discrimination at different eccentricities" Journal of the Optical Society of America A 4 1699-1703

Schein S J, 1988 "Anatomy of macaque fovea and spatial densities of neurons in foveal representations" Journal of Comparative Neurology $269479-505$

Schein S J, De Monasterio F M, 1987 "Mapping of retinal and geniculate neurons onto striate cortex of macaque" Journal of Neuroscience 7 996-1009

Schwartz E L, 1980 "Computational anatomy and functional architecture of striate cortex: A spatial mapping approach to perceptual coding" Vision Research 20 645-669

Schwartz E L, 1984 "Spatial mapping and spatial vision in primate striate and infero-temporal cortex", in Sensory Experience, Adaptation, and Perception Eds L Spillmann, B R Wooten (Hillsdale, NJ: Lawrence Erlbaum Associates) pp 73-104

Schwartz E L, 1994 "Computational studies of the spatial architecture of primate visual cortex", in Cerebral Cortex volume 10 Primary Visual Cortex in Primates Eds A Peters, K S Rockland (New York: Plenum Press) pp 359-411

Sereno M I, Dale A M, Reppas J B, Kwong K K, et al, 1995 "Borders of multiple visual areas in humans revealed by functional magnetic resonance imaging" Science 268 889-893

Teuber H L, Battersby N S, Bender M F, 1960 Visual Field Defects after Penetrating Missile Wounds of the Brain (Cambridge, MA: Harvard University Press)

Tootell R B H, Silverman M S, Switkes E, De Valois R L, 1982 "Deoxyglucose analysis of retinotopic organization in primate striate cortex" Science $218902-904$ 
Tootell R B H, Switkes E, Silverman M S, Hamilton S L, 1988 "Functional anatomy of macaque striate cortex. II. Retinotopic organization" Journal of Neuroscience 81531 - 1568

Van Essen D C, Newsome W T, Maunsell J H R, 1984 "The visual field representation in striate cortex of the macaque monkey: Asymmetries, anisotropies, and individual variability" Vision Research $24429-448$

Virsu V, Rovamo J, 1979 "Visual resolution, contrast sensitivity and the cortical magnification factor" Experimental Brain Research 37 475-494

Virsu V, Rovamo J, Laurinen P, Näsänen R, 1982 "Temporal contrast sensitivity and cortical magnification" Vision Research $221211-1217$

Wässle H, Grunert U, Rohrenbeck J, Boycott B B, 1989 "Cortical magnification factor and the ganglion cell density in the primate retina" Nature (London) 341 643-646

Watson A B, 1983 "Detection and recognition of simple spatial forms", in Physical and Biological Processing of Images Eds O J Braddick, A C Sleigh (New York: Springer)

Watt R J, Morgan M J, 1983 "The recognition and representation of edge blur: Evidence for spatial primitives in human vision" Vision Research 23 1465-1477

Wertheim T, 1980 "Peripheral visual acuity" American Journal of Optometry 57 915-934

Westheimer G, 1967 "Spatial interaction in human cone vision" Journal of Physiology (London) $190139-154$

Westheimer G, 1991 "Sharpness discrimination for foveal targets" Journal of the Optical Society of America A 8 681-685

Weymouth F, 1958 "Visual sensory units and the minimal angle of resolution" American Journal of Ophthalmology $46102-113$

Wilson H R, Levi D, Maffei L, Rovamo J, De Valois R, 1990 "The perception of form: Retina to striate cortex", in Visual Perception, the Neurophysiological Foundations Eds L Spillmann, J S Werner (San Diego, CA: Academic Press) pp 231-272

Wright M J, Johnston A, 1985 "Invariant tuning of motion aftereffect" Vision Research 25 1947-1955 